Pathologe $2021 \cdot 42: 224-230$

https://doi.org/10.1007/s00292-020-00892-8

Angenommen: 15. Dezember 2020

Online publiziert: 12. Januar 2021

(c) Springer Medizin Verlag $\mathrm{GmbH}$, ein Teil von Springer Nature 2021

\section{Schwerpunktherausgeber}

W. Roth, Mainz

P. Boor, Aachen

Pandemien zeigen wiederkehrende, gemeinsame Charakteristika. Zum einen das Ad-hoc-Auftreten eines Erregers, beispielsweise durch eine plötzliche Verschleppung in einen neuen Lebensraum oder bei dem Sprung aus dem Tierreservoir auf den Menschen, zum anderen die damit verbundene Unsicherheit aufgrund fehlender Daten, Konzepte und Erfahrungen. In einem von Mangel an gesichertem Wissen geprägten Umfeld liegt daher die Hoffnung auf der Wissenschaft, diese Wissenslücken zu füllen und so zu einer Krisenbewältigung beizutragen - wie auch bei COVID19.

Zur Eindämmung von Epidemien und Pandemien stellt die Wissenschaft nicht nur Daten für das öffentliche Gesundheitswesen, sondern auch für die Politik und ihre Berater zur Verfügung, um maßgeschneiderte Richtlinien, Verordnungen und Gesetze zum Schutze der Gesellschaft erlassen und eine effektive (nationale) Bewältigungsstrategie festlegen zu können. Sinnvolle Präventionsmaßnahmen lassen sich nur dann anordnen, wenn Faktoren wie Fallzahlen, deren Clustering, mögliche Übertragungswege oder die Reproduktionszahl eines Erregers bekannt sind. Zudem braucht es ein allgemeines Verständnis einer Krankheit, zum Beispiel bezüglich der möglichen Risikogruppen und deren Schutz, um wissenschaftliche Erkenntnisse in eine sinnvolle Kommunikation mit der Öffentlichkeit $\mathrm{zu}$ integrieren, deren Einsatz und Engagement einen Kernbestandteil einer

Stephanie Strobl · Wilfried Roth

Institut für Pathologie, Universitätsmedizin Mainz, Johannes Gutenberg-Universität Mainz, Mainz, Deutschland

\title{
Internationale wissenschaftliche Publikationsaktivität zu COVID-19
}

Bewältigungsstrategie darstellen. Letztlich wird neues Wissen auch für die Wissenschaft selbst generiert, da grundlegende Erkenntnisse als Basis für weiterführende, spezifischere Forschung dienen, die wiederum Raum für mehr interdisziplinäre Kooperation lässt.

Gerade zu Beginn einer Krise, wie aktuell im Rahmen der COVID-19-Pandemie, steht demnach die Wissenschaft in der besonderen Verantwortung, die Basis für eine effektive nationale und internationale Bewältigung zu schaffen. Von der Norm abweichend ist in einer solchen Situation der zeitliche, aber auch politische Druck, der auf der Wissenschaft und ihren Beschäftigten lastet. In diesem Artikel soll daher zunächst analysiert werden, wie die wissenschaftliche Gemeinschaft publikatorisch aufdie akute Herausforderung der COVID-19-Pandemie reagiert hat. Ferner soll dargestellt werden, welche Rolle hierbei das Fachgebiet der Pathologie einnimmt. Schließlich wird diskutiert, wie COVID-19 das wissenschaftliche $\mathrm{Pu}$ blizieren weltweit verändert.

\section{Methodik}

Um die Anzahl der Publikationen zu bestimmen, verwendeten wir die Advanced-Search-Funktion von PubMed ${ }^{\circledR}$. Mithilfe individuell zusammengestellter Suchtermini ließ sich die Zahl der veröffentlichten Publikationen zum Thema COVID-19 (einschließlich der intermediären Bezeichnung nCov-19 und des Erregers SARS-CoV-2) auf Zeitraum und Art der Publikation sowie die Beteiligung der Ursprungsländer und des Fachbereiches der Pathologie bestimmen. Die Zeiträume grenzten wir jeweils vom ersten bis zum letzten Tag eines Monats ein, mit Beginn im Januar 2020, während wir manuell die Publikationen ausschlossen, die bereits elektronisch in früheren Monaten erschienen waren. Unter allen möglichen Filtern bezüglich der Publikationsarten wählten wir klinische Studien (einschließlich randomisiert-kontrollierter Studien und multizentrischen Studien), Reviews (einschließlich systematischen Reviews sowie Metaanalysen), Fallstudien, Editorials und Originalartikel aus, da diese den Publikationsformen mit der größten Relevanz für die klinische Praxis entsprechen. Bezüglich der Länderbeteiligung legten wir den Fokus auf die 10 führenden Nationen im Bereich der naturwissenschaftlichen Forschung entsprechend ihres Publikationsvolumens nach Nature Index [1]. Hierbei bezieht die Länderbeteiligung die Nationalitäten aller an der Publikation beteiligten Autoren mit ein, ohne dabei aus technischen Gründen eine Gewichtung nach beispielsweise Erst- und Letztautorenschaften vorzunehmen. Die Beteiligung der Pathologie wurde durch den Suchterminus ,affiliation“ berücksichtigt, der die gesamten Autoren und die für den Artikel gelisteten beteiligten Forscher mit Zugehörigkeit zu einem Institut für Pathologie einschließt. Zur Erfassung der Zahl der Neuinfektionen beziehen wir uns auf Daten der Onlineplattform Our World in Data der Universität Oxford, die zudem weitere Indizes bereitstellt, sowie auch Bevölkerungszahlen, um auf die Infektionszahlen pro 100.000 Einwohner zu normieren. 


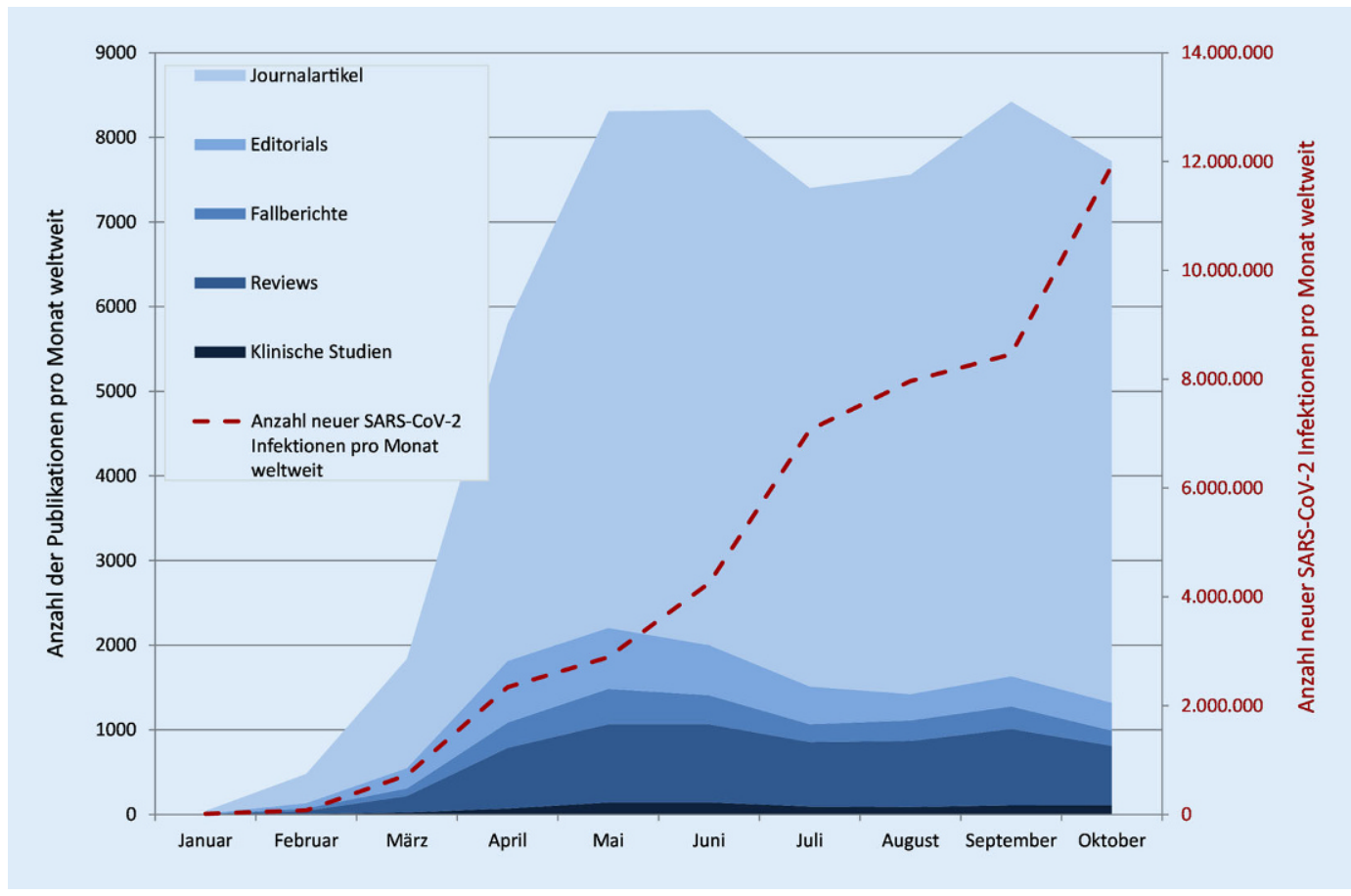

Abb. $1 \triangleleft$ Art der Publikationen pro Kalendermonat

\section{Publikationsaktivität in Zeiten von COVID-19}

Bei Betrachtung der Anzahl der Publikationen insgesamt zeigt sich zu Beginn ein Anstieg ähnlich des Anstiegs neuer SARS-CoV-2-Infektionen weltweit (• Abb. 1). Während die Zahl der Infektionen jedoch bis Ende Oktober in Wellen stetig weiter stieg, erreichten die Zahlen neuveröffentlichter Journalartikel, klinischer Studien, Editorials, Fallberichten und Reviews ihre Höhepunkte zu jeweils verschiedenen Zeitpunkten.

Einige Entwicklungen sind dabei gesondert hervorzuheben. So sank die Zahl der Editorials mit der Thematik COVID19 nach einem Höhepunkt im Mai. Etwa zur gleichen Zeit fiel auch die Zahl der Fallberichte, was möglicherweise damit erklärt werden kann, dass Fallstudien vor allem zu Beginn einer Krise wichtig sind, wenn noch keine größeren Kohortenstudien möglich sind. Erwartungsgemäß erreichten Journalartikel und klinische Studien ihren Höhepunkt geringfügig später, bevor auch hier die Zahl langsam abfiel. Diese Verzögerung ließe sich zum Beispiel mit dem etwas größeren Aufwand der Datenerfassung erklären. Besonders für die Journalartikel folgte dann ein weiterer Höhepunkt im September.
Insgesamt stieg jedoch nicht die Anzahl der medizinischen Publikationen an sich, sondern lediglich der Anteil COVID-19-bezogener Veröffentlichungen am gesamten Publikationsvolumen. Vergleichbar zu den bisherigen Ergebnissen steigerte sich der Anteil der auf COVID-19-bezogenen Publikationen bis Mai, bevor sich für jedes Land ein individuell stabiles Level einpendelte (beispielsweise für die USA bei $6 \%$, für China bei $5 \%$ und für Deutschland bei $4 \%$ des Gesamtvolumens).

Doch nicht nur das Publikationsvolumen, auch der Zeitraum zwischen Beginn der Krise und den beschriebenen Publikationsspitzen ist interessant. Davon ausgehend, dass COVID-19 vor allem Mitte bis Ende Februar 2020 begann, die Aufmerksamkeit auf sich zu ziehen, würde dies bedeuten, dass sich die Publikationshöhepunkte nur 3-4 Monate später abzeichneten, was für einen insgesamt sehr schnellen Prozess der Datenerhebung, der Auswertung und des Verfassens eines Artikels sprechen würde. Die Brisanz der Thematik spiegelt sich also durchaus in der außerordentlichen Schnelligkeit der Veröffentlichung wider. Dies lässt folglich 2 mögliche Interpretationen der sinkenden Publikationszahlen in den Monaten Juni und Juli zu.
Zum einen könnte der leichte Abfall des Publikationsvolumens damit erklärt sein, dass die klinischerseits wichtigsten Fragen in Bezug auf Inhalte wie Transmission und Krankheitsverlauf von SARSCoV-2-Infektionen bereits - zumindest partiell - beantwortet waren, was wiederum die zeitliche Dringlichkeit und den Neuheitscharakter der Publikationen abmilderte. Zum anderen könnte die geringere Anzahl an Publikationen den Daten entsprechen, die im Juli bezüglich einer neuen oder spezifischeren Fragestellung erhoben wurden und noch zur Veröffentlichung ausstehen. Die zum September wieder leicht steigenden Publikationszahlen unterstreichen diese These.

Entsprechend stellt sich außerdem die Frage, ob die Anzahl der Publikationen in einem direkten Zusammenhang mit der Zahl der Neuinfektionen der Länder steht, da es vorstellbar wäre, dass eine größere Zahl an SARS-CoV-2-Infektionen potenziell zu mehr Daten und damit zu mehr Publikationen führen könnte. Hierbei zeigte sich länderübergreifend, analog zu der vorherigen Betrachtung der Artikelarten, ein Spitzenwert für die Gesamtzahlen an Publikationen für die Monate Mai und Juni mit entsprechendem Abfall im Juli, wobei der Publikationshöhepunkt in einigen Ländern wie 
in China, Südkorea und Australien leicht früher einsetzte. Hierbei handelt es sich jedoch auch um Staaten, die bereits früh mit SARS-CoV-2-Infektionen konfrontiert waren (• Abb. 2). Insgesamt zeigt sich ein Höhepunkt an Publikationszahlen 1-2 Monate nach dem Spitzenwert für Neuinfektionen, ohne eine klar erkennbare quantitative Assoziation zwischen Ausmaß der Publikationsaktivität und Ausmaß der Ausbreitung der Infektion im nationalen Vergleich.

Bezüglich des Publikationsvolumens ergeben sich ebenfalls interessante $\mathrm{Be}$ funde. Während sich die USA erwartungsgemäß an vorderer Stelle wiederfinden, zeigten Japan und Südkorea eher geringe Publikationsaktivität, obwohl bereits im Januar SARS-CoV-2-Infektionen gemeldet wurden. Die Verbreitung des Erregers konnte jedoch hier auch zeitnah eingedämmt werden. Australien hingegen, in einer ähnlichen Situation, zeigte eine deutlich höhere Publikationsaktivität. Bemerkenswert ist auch die hohe Anzahl von Publikationen aus China bei offenbar in den letzten Monaten sehr geringen Infektionszahlen.

Die Beteiligung der Pathologie zeigt sich im Verlauf der Pandemie als tendenziell steigend ( $\bullet$ Abb. 3). Dies ist ein weiteres Anzeichen dafür, dass im Verlauf des Wissensgewinns die Fragestellungen spezifischer werden.

\section{Publizieren in Krisenzeiten: Chancen und Risiken}

Anhand dieser Daten zeichnen sich bestimmte Besonderheiten für das wissenschaftliche Arbeiten zu Beginn und während der COVID-19-Pandemie ab. Wenig überraschend ist dabei die verkürzte Zeitspanne zwischen Datenerhebung und Publikation. Kontraintuitiv erscheint jedoch, dass sich die bisher geltende geografische Hierarchie im Bereich naturwissenschaftlicher Publikationen nicht vollständig auf die Aktivität der Forschung zu SARS-CoV-2 widerspiegelt. COVID-19 scheint also bereits die aktuelle Publikationslandschaft verändert zu haben.

Am offensichtlichsten ist hierbei die hohe Anzahl an Publikationen zum Thema SARS-CoV-2. Zum Vergleich: Be-

Pathologe 2021 - 42:224-230 https://doi.org/10.1007/s00292-020-00892-8

(c) Springer Medizin Verlag GmbH, ein Teil von Springer Nature 2021

\section{S. Strobl · W. Roth}

\section{Internationale wissenschaftliche Publikationsaktivität zu COVID-19}

\section{Zusammenfassung}

Hintergrund. Pandemien führen zu neuen Herausforderungen für das Gesundheitssystem und damit zu einer Verschiebung des Fokus und der Arbeitsweise in der Wissenschaft, was sich wiederum auf die globale Publikationsaktivität auswirkt. Fragestellung. Die Entwicklung der Publikationsaktivität im Verlauf der COVID-19Pandemie im internationalen Vergleich sowie in Bezug auf lokale Infektionszahlen und die Beteiligung des Fachgebiets der Pathologie. Material und Methode. Vergleichende Analyse der Anzahl der Publikation der Metadatenbank PubMed ${ }^{\circledR}$ zum Thema COVID-19 bezüglich der Artikelart, des Publikationszeitraums und -ortes, der Zugehörigkeit der Autoren zu einem Institut für Pathologie sowie der Korrelation mit den jeweiligen Infektionsfallzahlen des gleichen Zeitraums.

Ergebnisse. Es zeigt sich ein Publikationshöhepunkt im Mai/Juni 2020 mit anschließendem leichten Abfall der Publikationszahlen sowie einem erneuten
Anstieg ab August/September 2020. Zudem verkürzte sich die Zeitspanne zwischen Datenerhebung und Veröffentlichung auf etwa 3-4 Monate. Länder mit frühzeitigem Erstkontakt mit SARS-CoV-2 publizierten zeitnah, bei jedoch fehlender Korrelation der Anzahl der Publikationen mit COVID-19Fallzahlen. Die Beteiligung des Fachgebiets der Pathologie lag bei durchschnittlich 4\% der beitragenden Autoren und nahm im Verlauf der Pandemie zu.

Schlussfolgerungen. Die globale Publikationsaktivität zu COVID-19 ist geprägt durch eine außergewöhnlich hohe Anzahl an Publikationen und eine verhältnismäßig kurze Zeitdauer der Forschungsvorhaben, unabhängig von Ursprungsland und Höhe der Infektionszahlen in der Bevölkerung.

\section{Schlüsselwörter}

COVID-19. Publikationsaktivität $\cdot$ Krisenreaktion · Krisenbewältigung · Wissenschaftliches Publizieren

\section{International publication activity during the COVID-19 pandemic}

\section{Abstract}

Background. Pandemics lead to new challenges for healthcare systems and a subsequent shift of the scientific focus, which can partially be seen in alterations in scientific publication activity.

Objectives. Follow-up on the global publication activity within the course of the COVID-19 pandemic in a comparison of national contexts with regards to local infection rates and the involvement of the discipline of pathology.

Materials and methods. Comparative analysis of the number of publications in the PubMed ${ }^{\circledR}$ database concerning COVID19 with respect to publication type, date and place of publication, affiliation to an institute of pathology, and correlation with the number of SARS-CoV-2 infections over the same timeframe.

Results. After an initial peak with regards to the number of publications in the months of May and June 2020, a slight decrease was observed, followed by another increase starting in August/September 2020. Further, the time between data collection and publication contracted to approximately 3-4 months. Countries faced with early SARSCoV-2 infections published promptly, even though there was no overall association between the number of publications and COVID-19 case numbers. On average, 4\% of authors were affiliated to an institute of pathology, with a steady increase of this percentage within the course of the pandemic.

Conclusions. COVID-19 altered global publication activity by providing for an unprecedented number of publications combined with an acceleration of publication times irrespective of the geographical location and overall case numbers.

\section{Keywords} COVID-19 · Publication activity · Crisis response $\cdot$ Crisis management $\cdot$ Scientific publishing 


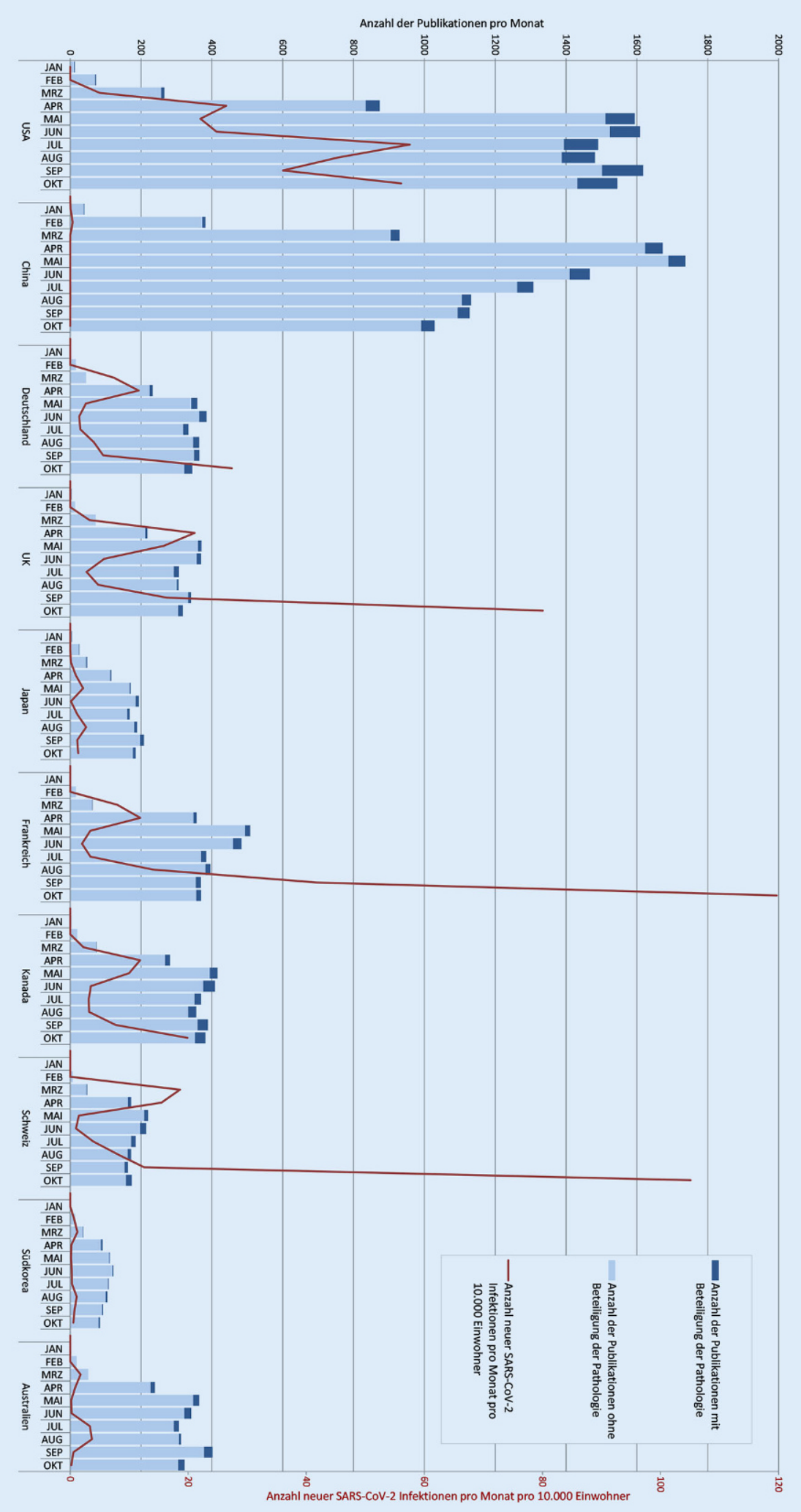

Abb. $2 \triangle$ Anzahl der Publikationen pro Kalendermonat und Land züglich des Ausbruchs des Zika-Virus in Lateinamerika im Januar 2016, der wie COVID-19 von der WHO zu einer gesundheitlichen Notlage internationaler Tragweite erklärt wurde, sind für die Monate Januar bis Juli 2016 lediglich 644 Publikationen auf PubMed ${ }^{\circledR} \mathrm{zu}$ verzeichnen - gegenüber 41.525 Veröffentlichungen bezüglich COVID-19 im gleichen Zeitraum im Jahr 2020. Keine Epidemie oder Pandemie zuvor hat demnach ein solches öffentliches als auch wissenschaftliches Interesse auf sich ziehen können, was selbstverständlich nicht zuletzt vor allem auf die Schwere und das Ausmaß der COVID-19-Pandemie mitsamt ihrer ökonomischen Folgen zurückzuführen ist.

Jedoch ist nicht nur die Anzahl der veröffentlichten Publikationen präzedenzlos, sondern auch die erheblich verkürzte Zeitspanne zwischen Datenerhebung und Veröffentlichung einer Publikation. Dieses beschleunigte Publikationsverfahren wird vor allem durch einen beschleunigten Peer-Review-Prozess ermöglicht. Zu diesem Schluss kam eine Studie des Instituts für Wissenschaft und Technologie der Universität Leiden, die den Reviewprozess von 14 medizinischen Journalen untersuchte. Die Prozessierungszeit der Fachzeitschriften verringerte sich gemäß dieser Studie um eindrückliche $49 \%$ [2].

Die Bereitstellung von Publikationen befindet sich, angestoßen durch die Forschung zu SARS-CoV-2, ebenfalls im Wandel. Hierbei scheint sich auch für den medizinischen Bereich eine neue Art der Veröffentlichung zu etablieren, die Mathematiker und Physiker bereits seit geraumer Zeit nutzen. Auf sogenannten Preprintservern werden dabei Inhalte geteilt ohne dass ein zeitaufwendiges PeerReview-Verfahren durchlaufen werden muss. Schätzungen zufolge wurden im Rahmen der COVID-19-Pandemie bereits etwa die Hälfte der Arbeiten auf besagten Servern veröffentlicht [3].

Ein weiteres Novum zeigt sich in der Bereitschaft der Verlage, Publikationen zum Thema COVID-19 frei zugänglich zur Verfügung zu stellen. Doch nicht nur Verlage, sondern auch internationale Organisationen signalisieren ihre volle Unterstützung für die öffentliche Be- 




Abb. $3 \Delta$ Die Beteiligung der Pathologie in Bezug auf das gesamte Publikationsvolumen reitstellung wissenschaftlicher Arbeiten, wie beispielsweise die UNESCO und ihre Partner, die die Wichtigkeit von „open access and open data "für eine bessere und schnellere Forschung unterstreichen [4]. Diese Aufforderung wurde von der Wissenschaft offensichtlich wahrgenommen. Eine Studie der Universität Madrid zeigt, dass 97,4\% der SARS-CoV-2 betreffenden Publikationen auf PubMed ${ }^{\circledR}$ frei zugänglich sind [5]. Forscher der Universität Granada kommen in einer ähnlichen Arbeit auf lediglich 67,5\% [6].

Ein einfacher Zugang zu neuen Erkenntnissen ist jedoch nicht nur für die Wissenschaft, sondern auch für die $\mathrm{Ge}$ sellschaft relevant. Wie eine Studie der Universität Amsterdam zeigte, liegen den auf COVID-19 bezogenen Artikeln der Plattform Wikipedia vorrangig wissenschaftliche Publikationen zugrunde, die einem Peer-Review-Prozess standhielten [7]. Die Wichtigkeit, einer breiten Öffentlichkeit leicht zugängliche und vor allem verständliche Informationen zur Verfügung stellen zu können beruht dabei jedoch nicht nur darauf, dass das öffentliche Engagement eine Grundlage für eine effektive Bewältigungsstrategie darstellt, sondern auch, um der Verbreitung von Fehlinformationen und Falschmeldungen, zum Beispiel über sozialen $\mathrm{Me}$ dien, entgegenzuwirken.

Auch wenn zu Beginn eines Ausbruchs einer Infektionskrankheit schnelles Handeln essenziell ist, bergen einige dieser Entwicklungen erhebliche Risiken. Veröffentlichung ohne vorhergehende Qualitätskontrolle des Inhalts, beispielsweise durch ein Peer-Review-Verfahren, lassen Raum für eine Verbreitung von Fehlinformationen und Arbeiten auf Basis schlechter wissenschaftlicher Praxis. Im Januar 2020 veröffentlichte beispielsweise ein indischer Forscher eine wissenschaftlich nicht haltbare Theorie über einen mutmaßlichen Zusammenhang zwischen den Erregern SARSCoV-2 und HIV, die widerrufen wurde, nachdem sich mehrere Wissenschaftler einschalteten, um auf methodologische Fehler hinzuweisen [3]. Dieses Beispiel macht deutlich, welche schwerwiegenden Konsequenzen es haben könnte, wenn pseudowissenschaftliche Arbeiten dieser Art über einen längeren Zeitraum 
kursieren würden und damit beispielsweise für die Öffentlichkeit und Politik zugänglich wären, die möglicherweise nicht über die nötige Vorbildung verfügen, um solche Artikel folgerichtig bewerten zu können. Gleichwohl sind auch in Fachzeitschriften veröffentliche Artikel nicht gänzlich vor Fehlern gefeit. Die im Nachhinein wissenschaftlich wohl nicht begründbare Idee, Hydroxychloroquin als mögliche Therapie für COVID-19 einzusetzen, wurde beispielsweise durch eine Arbeit französischer Wissenschaftler im International Journal of Antimicrobial Agents gestützt, die einen Peer-Review-Prozess überstanden hatte [3].

Eine weitere Herausforderung besteht darin, dass Ärzten und Wissenschaftlern durch die Flut neuer Daten und Ergebnissen die Übersicht genommen wird. Alleine im Mai wurden beispielsweise innerhalb nur einer Woche über 4000 neue Publikationen veröffentlicht [8]. Einen Lösungsansatz bieten Datenportale, wie beispielsweise das COVID-19 Open Research Dataset (CORD-19), die Artikel hochrangiger Journale und Arbeiten von Preprintservern bündeln [9]. Durch verschiedenste Algorithmen werden Artikel mit ähnlichen Inhalten markiert, während mithilfe von Filteroptionen Ärzte und Forscher eine auf persönliche Interessen eingeschränkte Auswahl an Arbeiten zusammenstellen können.

Nicht zu vergessen ist auch die Tatsache, dass sich die zuvor beschriebene verkürzte Zeit zwischen Einreichung und Publikation lediglich auf Artikel bezog, die die COVID-19-Pandemie betrafen [2]. Hierbei stellt sich die Frage, ob durch den Fokus aufSARS-CoV-2 andere wichtige Themen von der wissenschaftlichen Bildfläche verdrängt werden bzw. deren Publikation verzögert wurde. Dies äußert sich zum Beispiel auch darin, dass sich viele Wissenschaftler von ihren ursprünglichen Themengebieten abwendeten, um sich SARS-CoV-2-verwandten Themen zu widmen [10].

\section{Die Wissenschaft Post-COVID: Ein Ausblick}

Insgesamt zeigt die COVID-19-Pandemie, dass sich die wissenschaftliche
Arbeit nicht nur verändert, sondern auch durch kreative Ideen zur Begegnung aufkommender Herausforderungen bereichert wird. Ein weiteres Beispiele hierfür ist die Initiative Review Commons, ein Zusammenschluss verschiedener Forscher und Wissenschaftler, die sich bereit erklärt haben, an einem beschleunigten Peer-Review-Prozess teilzunehmen [10], um zu ermöglichen, dass schnell publiziert werden kann, ohne qualitative Einbußen in Kauf nehmen zu müssen. Mit einem ähnlichen Prinzip arbeitet die Initiative Rapid Reviews: COVID-19 der Universität Berkeley und MIT Press.

Während einige dieser Veränderungen möglicherweise vergänglich sind, sobald das Publikationsvolumen weiter absinkt, mögen andere über den Verlauf der Krise erhalten bleiben. So bestätigt John Inglis, Mitbegründer der Preprintserver medRxiv, gesponsort durch die Universität Yale, und bioRxiv, unterstützt durch die Non-Profit-Organisation Cold Spring Harbor Laboratory, dass wissenschaftliche Zweige, die besagte Server nutzen, für gewöhnlich nicht mehr davon abkehren [11]. Ein ähnliches Phänomen wird im Bereich der Medikamentenzulassung erwartet. Während die Bereitschaft der zuständigen Behörden, eine schnelle und unkomplizierte Zulassung von Studien oder Medikamenten zur Erforschung und Behandlung von COVID19 zu ermöglichen, auf die Pandemie beschränkt sein wird, besteht die Hoffnung, dass der Mut zu teils unüblichen Kollaborationen verschiedener Akteure, seien es Regierungen, die Industrie oder die akademische Welt, erhalten bleiben wird [12]. Auch besteht die Hoffnung, dass sich die Onlinelehre nachdrücklich verbessert und sich zukünftig inklusiver gestaltet - sowohl im globalen Norden als auch in Entwicklungsländern, die letztlich nun zu einer Erweiterung ihrer digitalen Infrastruktur gezwungen sind [13]. Wie sich dabei die erwarteten finanziellen Schwierigkeiten auswirken werden, bleibt unabhängig des Herkunftslandes abzuwarten.

Ein Hoffnungsschimmer ist dabei, dass die Wissenschaft von ursprünglichen Fehlern gelernt zu haben scheint. So veröffentlichte das WHO Bulletin noch 2016 einen Aufruf zu einer schnelleren, transparenteren und umfassenderen Veröffentlichung von Forschungsergebnissen, da eine fehlende Initiative in diesem Bereich die Antwort auf die Ebola-Epidemie in Westafrika in 2014 merklich verlangsamte [14]. Auch wenn das Interesse und die Bereitschaft zur Kooperation westlicher Industrienationen während der COVID-19-Pandemie vermutlich besonders auf die wirtschaftliche und politische Tragweite ihrer Konsequenzen zurückzuführen ist, bleibt $\mathrm{zu}$ hoffen, dass der globale Einsatz für eine weitreichende wissenschaftlichen Zusammenarbeit für die Zeit nach der Krise erhalten bleibt. Dies zu beweisen gilt es in den Epidemien und Pandemien, die noch vor uns liegen.

\section{Fazit für die Praxis}

- Die Anzahl der Publikationen zum Thema COVID-19 ist präzedenzlos und kontinuierlich weiter steigend, bei einem zwischenzeitlichen leichten Abfall in den Monaten Mai/Juni 2020. Diese Entwicklungen sind unabhängig von Ursprungsland, der Höhe der Infektionszahlen und der bisherigen Publikationsleistungen.

- Die Zeitspanne zwischen Datenerhebung und Veröffentlichung COVID-19-assoziierter Publikationen verkürzte sich dabei auf etwa 3-4 Monate.

- Die Beteiligung des Fachgebiets der Pathologie lag bei durchschnittlichen $4 \%$ der beitragenden Autoren und nahm im Verlauf der Pandemie aufgrund spezifischerer Fragestellungen zu.

- Es etablieren sich neue Praktiken des wissenschaftlichen Publizierens, zum Beispiel durch die Nutzung von Preprintservern, Initiativen für beschleunigte Peer-ReviewVerfahren sowie durch neue, frei zugängliche Datenportale für die Wissenschaft und Öffentlichkeit. 


\section{Korrespondenzadresse}

Dr. med. Stephanie Strobl,
Master of Advanced
International Studies
(M.A.I.S.)
Institut für Pathologie,
Universitätsmedizin Mainz,
Johannes Gutenberg-
Universität Mainz
Langenbeckstr. 1,
55131 Mainz, Deutschland
stephanie.strobl@unimedizin-
mainz.de

\section{Einhaltung ethischer Richtlinien}

Interessenkonflikt. S. Strobl und W. Roth geben an, dass kein Interessenkonflikt besteht.

Für diesen Beitrag wurden von den Autoren keine Studien an Menschen oder Tieren durchgeführt. Für die aufgeführten Studien gelten die jeweils dort angegebenen ethischen Richtlinien.

\section{Literatur}

1. Nature Index (2020) The ten leading countries in natural-sciences research. https://www.nature. com/articles/d41586-020-01231-w.Zugegriffen: 7. Sept. 2020 (Nature Index 2020 Annual Tables)

2. Horbach S (2020) Pandemic Publishing: Medical journals drastically speed up their publication process for Covid-19. bioRxiv. Institute for Science in Society, Faculty of Science, Radboud University, Nijmegen

3. The Economist (2020) Scientific research on the coronavirus is being released in a torrent. https://www.economist.com/science-andtechnology/2020/05/07/scientific-research-onthe-coronavirus-is-being-released-in-a-torrent. Zugegriffen: 15 . Sept. 2020

4. UNESCO (2020) Open access to facilitate research and information on COVID-19. https://en.unesco.org/covid19/ communicationinformationresponse/opensolutions. Zugegriffen: 15. Sept. 2020

5. Arrizabalaga O, Otaegui D, Vergara I, Arrizabalaga J, Mendez E (2020) Open Access of COVID-19related publications in the first quarter of 2020: a preliminary study based in PubMed. B.H.R. Institute, San Sebastian

6. Torres-Salinas D, Robinson-Garcia N, CastilloValdivieso PA (2020) Open Access and Altmetrics in the pandemic age: Forescast analysis on COVID-19 literature. D.d.I.y. Comunicación, Granada

7. Colavizza G (2020) COVID-19 research in Wikipedia. U.o. Amsterdam, Amsterdam

8. Brainard J (2020) Scientists are drowning in COVID19 papers. Can new tools keep them afloat? https:// www.sciencemag.org/news/2020/05/scientistsare-drowning-covid-19-papers-can-new-toolskeep-them-afloat. Zugegriffen: 15 . Sept. 2020

9. Hao K (2020) Over 24,000 coronavirus research papers are now available in one place. https://www.technologyreview.com/2020/03/ 16/905290/coronavirus-24000-research-papersavailable-open-data/.Zugegriffen: 15. Sept. 2020
10. Gibney E (2020) The pandemic mixed up what scientists study - and some won't go back. https:// www.nature.com/articles/d41586-020-01525-z. Zugegriffen: 15.Sept. 2020 (nature news feature)

11. Callaway $E$ (2020) Will the pandemic permanently alter scientific publishing? https://www.nature. com/articles/d41586-020-01520-4. Zugegriffen: 15. Sept. 2020 (nature news feature)

12. Ledford $H$ (2020) The coronavirus outbreak could make it quicker and easier to trial drugs. https:// www.nature.com/articles/d41586-020-01524-0. Zugegriffen: 15. Sept. 2020 (nature news feature)

13. Witze $A$ (2020) Universities will never be the same after the coronavirus crisis. https://www.nature. com/articles/d41586-020-01518-y. Zugegriffen: 15. Sept. 2020 (nature news feature)

14. Dye C, Bartolomeos K, Moorthy V, Kieny MP (2016) Data sharing in publichealth emergencies: a call to researchers. Bull World Health Organ 94(3):158

\section{Mit Stammzellen aus Fettge- webe Knochen heilen}

Herbert-Lauterbach-Preis 2020

Für seine Forschungen zur Knochenregeneration mithilfe von Stammzellen erhält der plastische Chirurg Prof. Dr. Björn Behr den Wissenschaftspreis der BG Kliniken.

Prof. Dr. Björn Behr erforscht am BG Universitätsklinikum Bergmannsheil die regenerativen Eigenschaften von Stammzellen. In seiner jüngsten Arbeit untersucht er das optimale Mischungsverhältnis von humanen Stammzellen aus Fettgewebe und Spenderknochen, um die Knochenheilung anzuregen. Dafür wurde er mit dem Herbert-Lauterbach-Preis der BG Kliniken ausgezeichnet.

Stammzellen können auf verschiedene Weise aus dem Körper eines Patienten gewonnen werden, besonders zahlreich treten sie im Fettgewebe auf. Mithilfe menschlicher Spenderknochen werden die Stammzellen in den Körper implantiert und der Knochendefekt aufgefüllt. Prof. Dr. Björn Behrs Studie zeigt, dass ein Mischungsverhältnis von 75.000 bis 100.000 Stammzellen pro 100 Kubikmillimeter Spenderknochen dabei die größten Effekte erzielt: Die Knochenregeneration und Gefäßneubildung der besiedelten Allografts verbesserte sich im Vergleich zum unbesiedelten Kontrollmodell deutlich, eine weitere Erhöhung des Anteils der eingebrachten Stammzellen bewirkte dagegen keine Verbesserung.

Der von den BG Kliniken verliehene Herbert-Lauterbach-Preis würdigt wissenschaftliche Leistungen, deren Fokus auf der medizinischen Versorgung von Versicherten der Berufsgenossenschaften und Unfallkassen liegt. Er wurde im Jahr 1984 ins Leben gerufen und ist mit 15.000 Euro dotiert.

Quelle: BG Universitätsklinikum Bergmannsheil Bochum www.bergmannsheil.de 